\title{
REVIEW ARTICLE Trace Minerals and Heavy Metals: Implications in Prostate Cancer
}

\author{
SIA Shah \\ Dept of Surgery \& Cancer, Faculty of Medicine, Imperial College London \& \\ NHS Hammersmith Hospital, London, United Kigdom
}

\begin{abstract}
Dietary minerals and environmental heavy metals are modifiable factors that have been studied over the years for associations with prostate cancer risk. This review consideres the recent evidence in this field and concludes that the role of dietary minerals and environmental elements in prostate cancer progression or prevention is not yet clear and further research is needed to elaborate on the current knowledge.
\end{abstract}

Key Words : Prostate cancer, Minerals, Heavy Metals

\section{Introduction}

Prostate cancer (PC) is one of the most common malignancies affecting men, particularly in the developed world. By 2030, the global annual incidence of PC is predicted to rise to 1.7 million. PC incidence rates vary greatly worldwide, being highest in western countries and relatively low in Asia. However, PC incidence is rising rapidly in Asia and factors responsible for this rapid rise include aging population, westernized dietary habits and increasing use of prostate-specific antigen (PSA) testing ${ }^{1,2}$.

Prostate tumors are predominantly adenoc arcinomas, having a heterogeneous and multifocal nature, and multiple mechanisms have been proposed for their initiation and progression $^{3}$. A salient aspect of PC disease process is its close association with aging; preneoplastic morphological changes are common in early life but progression to invasive carcinoma occurs late in a limited population as a consequence of aging. Familial inheritance is generally associated with early onset of disease and accounts for about $10 \%$ of $\mathrm{PC}^{4}$. Androgen receptor signaling has a central role in all stages of prostate carcinogenesis and transition to androgen independence is a hallmark feature of advanced disease ${ }^{5}$.

Bangladesh J Med Biochem 2015; 8(1): 27-32
In addition to these somewhat established pathophysiologic causes, diverse environmental factors including trace minerals and heavy metals are presumed to play a key role in prostate carcinogenesis. These factors can affect various aspects of cancer development which involves genetic alterations and selection for cells with increasing ability for proliferation, survival, invasion and metastasis ${ }^{6}$. Diet and supplementation are modifiable factors that have been studied for associations with PC risk and over the past few years, substantial research, focusing particularly on PC chemoprevention, has been carried out in this respect ${ }^{7}$. The present review summarizes recent clinical evidence regarding dietary minerals, their supplements and other environmental heavy metals implicated in PC.

\section{Selenium}

The trace element selenium ( $\mathrm{Se})$ is present in foods like grains, meat, poultry, fish, eggs and dairy products. Se occurs in both organic and inorganic forms including selenomethionine, selenocysteine, selenate and selenite and its bioavailability depends upon the chemical form 8,9 . The organic form enters through the food chain via the consumption of plants grown in soil containing the inorganic form. Following 
Trace Minerals and Heavy Metals: Implications in Prostate Cancer metabolism, Se is incorporated into selenoproteins which protect cellular membranes from oxidative damage and are also involved in reproduction, thyroid activity, DNA synthesis, muscle function and several other metabolic functions ${ }^{10,11}$.

Recent evidence has suggested the role of Se in reducing the risk of a variety of malignancies. Proposed mechanisms for anti-cancer actions of Se include antioxidant protection, enhanced carcinogen detoxification, enhanced immune surveillance, modulation of cell cycle, inhibition of tumor cell invasion and inhibition of angiogenesis ${ }^{10}$. Several studies have shown evidence of a link between low selenium levels and higher PC incidence and it has been suggested that higher levels of Se may slow PC tumour progression ${ }^{11-13}$. Results from the Nutritional Prevention of Cancer (NPC) trial of $200 \mu \mathrm{g} /$ day Se showed two-third reduction in PC incidence among men with low baseline PSA values $(<4 \mathrm{ng} / \mathrm{mL})$ but no such reduction was seen in participants with higher values. Interestingly, only participants with low baseline plasma selenium concentrations had significant reductions in PC incidence ${ }^{14}$.

Data from the Health Professionals Follow-Up Study showed that high baseline Se levels reduce the risk of advanced PC by more than half ${ }^{15}$. Results from a recent prospective study showed that toenail $\mathrm{Se}$ was associated with a reduced risk of advanced $\mathrm{PC}^{16}$. The Selenium and Vitamin E Cancer Prevention Trial (SELECT) tested Se and vitamin E separately and in combination but neither had any effect on the risk of $\mathrm{PC}^{17}$. Results from the Prostate Cancer Preventive Trial revealed no evidence that dietary or supplemental intake of $\mathrm{Se}$ was associated with risk of $\mathrm{PC}^{18}$. These conflicting findings about the role of Se in preventing $\mathrm{PC}$ could possibly be due to the type of $\mathrm{Se}$ administered or genetic susceptibility to its effect. Further work is needed to determine the effective type and dosage of Se before any recommendation can be made for its use in the prevention of $\mathrm{PC}$ or otherwise.
Zinc

Zinc ( $\mathrm{Zn})$ is an essential trace element possessing antioxidant properties and is a component of many proteins involved in physiological regulation of apoptosis, transcription and cellular differentiation. Zinc is abundant in many food sources, including red meat and poultry, beans, nuts, seafood, and whole grains, and can also be found in fortified breakfast cereals ${ }^{11}$. Zn accumulates greatly in the normal prostatic tissue, up to 10 times as much as in other soft tissues. Intraprostatic $\mathrm{Zn}$ is known to inhibit the mitochondrial enzyme aconitase, thereby limiting citrate oxidation and subsequent energy generation via Krebs cycle. It has been suggested that malignant cells become energy efficient by completing the Krebs cycle following loss of this inhibition by low $\mathrm{Zn}$ levels ${ }^{19,20}$. Conversely, high tissue $\mathrm{Zn}$ enhances the activity of telomerase which could possibly increase PC risk since telomerase is involved in unrestricted tumor cell proliferation ${ }^{21}$.

Results from clinical studies regarding the role of $\mathrm{Zn}$ in PC are not clear. Data from the Prostate Cancer Prevention Trial showed that high dietary $\mathrm{Zn}$ intakes modestly reduced the risk of high-grade disease ${ }^{18}$. Epstein et al. observed a potentially reduced PC risk in a recent study which showed an association between high dietary intake of zinc and lower PC-specific mortality after diagnosis, particularly in localized disease 22 . On the contrary, data from the Health Professionals Follow-Up Study demonstrated an increased likelihood of advanced PC with $\mathrm{Zn}$ in a daily dosage of more than $100 \mathrm{mg} /$ day but dietary $\mathrm{Zn}$ intake was not associated with PC risk $^{19}$. In a case-control study, Gallus et al. found an association between high $\mathrm{Zn}$ intake and risk for advanced $\mathrm{PC}^{23}$. These contradictory findings warrant further clinical exploration to explicate the potential mechanisms of PC development or prevention as a result of varying $\mathrm{Zn}$ concentrations. 


\section{Calcium}

Calcium $(\mathrm{Ca})$ is an essential element for humans, available only through dietary sources. It is largely concentrated in the skeleton as calcium-phosphate complexes, serving to maintain bone strength and $\mathrm{Ca}$ homeostasis. Non-skeletal $\mathrm{Ca}$ which accounts for approximately one percent of the total is responsible for a wide range of biological activities, including cell signalling, muscle contraction and nerve impulse transmission ${ }^{24}$.

The role of $\mathrm{Ca}$ in $\mathrm{PC}$ may possibly be due to the regulation of tumor cell growth and apoptosis by intracellular calcium ${ }^{25}$. Moreover, it has been theorized that dietary $\mathrm{Ca}$ may indirectly increase PC risk and promote tumor growth by reducing circulating levels of the biologically active form of vitamin D (1,25-dihydroxy cholecalciferol) which inhibits the proliferation of prostate cells ${ }^{26}$. Several studies have demonstrated that a high calcium intake from foods and/or supplements is associated with increased PC risk. Data from the Health Professionals Follow-up Study showed a significant association between high-grade PC (Gleason score $>7$ ) and high $\mathrm{Ca}$ intake but a nonsignificant, inverse association was observed for well-differentiated, localized disease. Higher $\mathrm{Ca}$ intakes (1500 mg/day) were associated with a higher risk of advanced disease but not with low grade, organ-confined cancers ${ }^{27}$.

Findings from the European Prospective Investigation into Cancer and Nutrition (EPIC) study showed an increased risk of $\mathrm{PC}$ with high total dietary $\mathrm{Ca}$ intake and dairy $\mathrm{Ca}$ intake. No association was observed between non-dairy $\mathrm{Ca}$ intake and the risk for $\mathrm{PCa}^{28}$. In the Prostate Cancer Preventive Trial, $\mathrm{Ca}$ intake was associated with an increased risk of low grade PC but inversely associated with high grade disease ${ }^{18}$. Williams et al. in a recent study showed that dietary $\mathrm{Ca}$ is associated with lower risk for PC overall, particularly among black men, and lower risk for high-grade cancer among all men ${ }^{29}$. The considerable inconsistency in findings regarding the impact of $\mathrm{Ca}$ intake on PC development and progression warrants additional investigation.

\section{Iron}

Iron $(\mathrm{Fe})$ is the most prevalent metal in the body. Bio-availability of $\mathrm{Fe}$ is affected by various factors, such as dietary sources and $\mathrm{Fe}$ form (heme or non-heme). Heme Fe content varies between foods from 17 to $80 \%$ of total Fe content $^{30-32}$. Heme $\mathrm{Fe}$ is more easily absorbed than non-heme Fe but depending on cooking method, its concentrations in meat can vary due to conversion into non-heme $\mathrm{Fe}$. Heme $\mathrm{Fe}$ is transported via blood circulation to all body organs including the prostate ${ }^{33}$.

Being a transition metal, Fe has loosely bound electrons in the outer shell which facilitate the production of reactive oxygen species (ROS) such as highly reactive hydroxyl radicals. Thus Fe overload can increase oxidative stress and cause DNA breaks and oxidative damage. Heme Fe can cause extensive cellular harm by catalyzing the generation of free radicals and it is also implicated in increased endogenous formation of N-nitroso compounds (NOC) which are known carcinogens in multiple species ${ }^{32}$.

Results from the Carotene and Retinol Efficacy Trial (CARET) study showed a significantly increased risk of clinically aggressive PC with higher total dietary Fe but did not reveal any association with overall PC. Associations were stronger among men with low dietary intake of fruits and vegetables which are foods rich in anti-oxidants $^{33}$. Sinha et al. studied the risk of PC with dietary iron intake (total and heme) and found no association between total iron intake and the overall PC risk or advanced disease. However, heme iron intake showed an increased risk for advanced cancer ${ }^{34}$. High Fe content of hair and nails have been linked with increased risk of $\mathrm{PC}^{35}$. 
Once absorbed, $\mathrm{Cd}$ binds to metallothionein and

Copper $(\mathrm{Cu})$ modulates the activities of multiple enzymes, regulates the redox state, promotes angiogenesis and mediates cellular proliferation. As a result of these activities, $\mathrm{Cu}$ appears to play an important role in the carcinogenic process which is evident through the increase seen in $\mathrm{Cu}$ levels in cancerous tissues ${ }^{36}$. Previously, a study of serum $\mathrm{Cu}$ levels in $\mathrm{PC}$ showed no increase as compared to normal controls ${ }^{37}$. However, a recent study documented higher $\mathrm{Cu}$ levels in hair and nails of PC patients ${ }^{35}$.

\section{Magnesium}

Magnesium $(\mathrm{Mg})$ is the second most abundant intracellular cation in the body, involved with numerous biological activities particularly related to its interaction with $\mathrm{Ca}$. Levels of both these cations are regulated through competition for intestinal absorption and renal reabsorption and also via a negative feedback system ${ }^{38}$. It has been indicated that the physiologic effects of $\mathrm{Ca}$ are enhanced in $\mathrm{Mg}$ deficiency as they compete for intracellular membrane binding sites ${ }^{39}$. Serum $\mathrm{Mg}$ levels, and $\mathrm{Ca} / \mathrm{Mg}$ ratio have been shown to be associated with high-grade PC. $\mathrm{Mg}$ deficiency is linked with chronic inflammation, possibly due to the concurrent $\mathrm{Ca}$ levels, which may play a key role in the progression to prostate cancer ${ }^{38}$.

\section{Cadmium}

Cadmium (Cd) is recognized as a carcinogen based particularly on studies of occupationally exposed individuals having substantial exposure via the respiratory system 40 . Chronic exposure to $\mathrm{Cd}$ poses a threat to the general population as it has a widespread presence in the environment 41 . Dietary vegetables and cereals are the main source of environmental $\mathrm{Cd}$ while drinking water contributes only a very small percentage 42 .

is stored mainly in the kidneys, liver and other organs. Prostate tissue has also been identified as a target for $\mathrm{Cd}$ deposition ${ }^{43}$. It has a long biological half-life (10-30 years) in humans which may cause neoplastic transformations in various organs including prostate ${ }^{44}$.Association between $\mathrm{Cd}$ and PC among occupationally exposed men was shown previously but more recent and larger studies did not confirm those findings. In a recent population-based prospective study, dietary cadmium exposure was associated with a slightly increased overall risk of PC and for localized disease, the risk was more marked in smokers and lean men ${ }^{45}$.

\section{Conclusion}

The current evidence seems a long way off in terms of classifying the exact roles of modifiable factors such as dietary minerals and environmental elements for offering clinically effective strategies aimed at modulating the risk of PC. Results from future experimental and clinical research employing adequately powered prospective study designs may help to clarify the etiologic role and/or chemopreventive potential of mineral elements in PC.

\section{References}

1. Siegel R, Naishadham D. and Jemal A, Cancer statistics, 2012. CA Cancer J Clin 2012. 62(1): 10-29.

2. Ito K. Prostate cancer in Asian men. Nat Rev Urol 2014. 11(4): 197-212.

3. Abate-Shen C. and Shen MM, Molecular genetics of prostate cancer. Genes Dev 2000. 14: 2410-34.

4. Grönberg H, Prostate cancer epidemiology. The Lancet, 2003; 361(9360): 859-864.

5. Prehn, RT, On the prevention and therapy of prostate cancer by androgen administration. Cancer Research 1999. 59: (4161-4).

6. Cooper G.M. The Development and Causes of Cancer, in The Cell: A Molecular Approach, M.A. Sunderland, Editor. Sinauer Associates; 2000

7. Posadzki P, et al., Dietary supplements and prostate cancer: a systematic review of double-blind, placebocontrolled randomised clinical trials. Maturitas 2013; 75(2): 125-30.

8. Van Poppel H. and Tombal B. Chemoprevention of 
prostate cancer with nutrients and supplements. Cancer Manag Res 2011; 3: 91-100.

9. Gray MA. et al., Environmental exposure to trace elements and prostate cancer in three New Zealand ethnic groups. International Journal of Environmental Research and Public Health 2005; 2(3): 374-84.

10. Papp LV, et al, From selenium to selenoproteins: synthesis, identity, and their role in human health. Antioxid Redox Signal 2007; 9(7): 775-806.

11. Platz EA. and Helzlsouer KJ, Selenium, Zinc, and Prostate Cancer. Epidemiologic Reviews 2001; 23: 93-102.

12. Shah SIA, Naik SI, and Nadeem I, Role of plasma selenium and osteopontin in prostate cancer. Scientific Medicine 2009; 1.

13. Peters U, et al. Serum selenium and risk of prostate cancer-a nested case-control study. American Journal of Clinical Nutrition, 2007. 85: 209-17.

14. Clark LC, et al. Effects of selenium supplementation for cancer prevention in patients with carcinoma of the skin: A randomized controlled trial. JAMA 1996; 276(24): 1957-1963.

15. Yoshizawa K et al. Study of Prediagnostic Selenium Level in Toenails and the Risk of Advanced Prostate Cancer. Journal of the National Cancer Institute 1998; 90(16): 1219-1224.

16. Geybels MS et al. Advanced prostate cancer risk in relation to toenail selenium levels. J Natl Cancer Inst 2013; 105(18): 1394-401.

17. Lippman SM, et al, Effect of selenium and vitamin E on risk of prostate cancer and other cancers. The selenium and vitamin $\mathrm{E}$ cancer prevention trial (SELECT). JAMA 2009; 301: 39-51.

18. Kristal AR et al Diet Supplement Use, and Prostate Cancer Risk: Results From the Prostate Cancer Prevention Trial. American Journal of Epidemiology 2010; 172(5): 566-577.

19. Leitzmann MF, et al Zinc Supplement Use and Risk of Prostate Cancer. Journal of the National Cancer Institute 2003; 95(13); 1004-1007.

20. Platz EA and KJ. Helzlsouer, Selenium, zinc, and prostate cancer. Epidemiologic Reviews 2001; 23: 93-101.

21. Nemoto $\mathrm{K}$ et al. Modulation of telomerase activity by zinc in human prostatic and renal cancer cells.
Biochemical Pharmacology, 2000; 59(4): p. 401-405.

22. Epstein MM, et al. Dietary zinc and prostate cancer survival in a Swedish cohort. Am J Clin Nutr 2011; 93(3): $586-93$

23. Gallus S et al. Dietary Zinc and Prostate Cancer Risk: A Case-Control Study from Italy. European Urology 2007; 52(4): 1052-1057.

24. Peacock M, Calcium metabolism in health and disease. Clin J Am Soc Nephrol 2010; 5 Suppl 1: 23-30.

25. Legrand $\mathrm{G}$, et al. $\mathrm{Ca} 2+$ pools and cell growth. Evidence for sarcoendoplasmic $\mathrm{Ca} 2+-\mathrm{ATPases} 2 \mathrm{~B}$ involvement in human prostate cancer cell growth control. J Biol Chem 2001; 276(50): 47608-14

26. Bonjour JP, Chevalley T, and Fardellone P, Calcium intake and vitamin $\mathrm{D}$ metabolism and action, in healthy conditions and in prostate cancer. Br J Nutr, 2007; 97(4): 611-6.

27. Giovannucci E, et al. A prospective study of calcium intake and incident and fatal prostate cancer. Cancer Epidemiol Biomarkers Prev 2006; 15(2): 203-10.

28. Allen NE. et al. Animal foods, protein, calcium and prostate cancer risk: the European Prospective Investigation into Cancer and Nutrition. Br J Cancer, 2008; 98(9): 1574-81.

29. Williams DC. et al. Dietary calcium and risk for prostate cancer: a case-control Study among US veterans. Preventing Chronic Disease 2012; 9: 39.

30. Boontaveeyuwat $\mathrm{N}$ and Klunklin $\mathrm{S}$, The heme iron content of urban and rural Thai diets. J Med Assoc Thai 2001; 84: 1131-6.

31. Lopez MA, and Martos FC., Iron availability: An updated review. Int J Food Sci Nutr 2004; 55(8): 597-606.

32. Bae YJ et al. Dietary intake and serum levels of iron in relation to oxidative stress in breast cancer patients. Journal of clinical biochemistry and nutrition, 2009; 45: 355-60.

33. Choi $\mathrm{Y}$ et al. Iron intake, oxidative stress-related genes (MnSOD and MPO) and prostate cancer risk in CARET cohort. Carcinogenesis 2008; 29(5): 964-70.

34. Sinha R. et al. Meat and meat-related compounds and risk of prostate cancer in a large prospective cohort study in the United States. Am J Epidemiol 2009; 170(9): 1165-77.

35. Karimi $\mathrm{G}$ et al., Association between Trace Element and Heavy Metal Levels in Hair and Nail with 
Prostate Cancer. Asian Pacific Journal of Cancer Prevention, 2012; 13(9): p. 4249-4253.

36. Majumder $\mathrm{S}$, et al. The role of copper in drug-resistant murine and human tumors. Biometals, 2009; 22(2): 377-84.

37. Adaramoye OA. Akinloye O, and Olatunji IK. Trace elements and Vitamin E status in Nigerian patients with prostate cancer. African Health Sciences 2010; 10(1): 1-8.

38. Dai Q et al. Blood magnesium, and the interaction with calcium, on the risk of high-grade prostate cancer. PLoS One 2011; 6(4): e18237.

39. Iseri LT and JH. French, Magnesium: Nature's physiologic calcium blocker. American Heart Journal, 1984; 108(1): 188-193.

40. Cho YE et al. Dietary cadmium intake and the risk of cancer: a aeta-analysis. Plos One, 2013; 8(9): e75087.
41. Jarup L. and Akesson A, Current status of cadmium as an environmental health problem. Toxicol Appl Pharmacol 2009; 238(3): 201-8.

42. Olsson I et al. Cadmium in blood and urine-impact of sex, age, dietary intake, iron status, and former smoking-association of renal effects. Environmental Health Perspectives 2002; 110: 1185-90.

43. Brys $M$ et al. Zinc and cadmium analysis in human prostate neoplasms. Biol Trace Elem Res 199;. 59: 145-52.

44. Waalkes MP, Cadmium carcinogenesis. Mutat Res 2003; 533: (107-20).

45. Julin B et al. Dietary cadmium exposure and prostate cancer incidence: a population-based prospective cohort study. Br J Cancer 2012; 107(5): 895-900. 